\title{
Factors affecting pitch judgments as a function of spectral composition
}

\author{
E. TERHARDT and A. GRUBERT \\ Institute for Electroacoustics, Technical University München, Munich, West Germany
}

\begin{abstract}
Stimulated by a recent paper by Platt and Racine (1985), we discuss the factors that probably are involved in certain inconsistencies observed in pitch judgments of tones with different spectral composition. Typically, discrepancies reported in the literature are of the order of 10 cents in magnitude. We point out that measurement of such small pitch effects is heavily dependent on systematic individual differences, and that, when individual differences are averaged out (as is essentially the case in Platt and Racine's experiments), verification of the actual auditory stimulus sound pressure level (SPL) within a few decibels is necessary. Utilizing the virtual-pitch theory, we evaluate the effects of frequency, SPL, and earphone-frequency response. Furthermore, we present experimental and theoretical data on pitch of piano tones relevant to the problem. The study elucidates that, taking into account the factors mentioned, agreement between the various data considered, as well as theoretical understanding, actually is much better than would be apparent at first sight.
\end{abstract}

Recently, Platt and Racine (1985) provided further experimental data on small, but systematic, differences in the judgment of the pitch of tones having different spectral composition (see the literature listed by Platt and Racine). In particular, the authors rightly drew attention to the fact that observations reported by different authors are more or less variable, and even appear to be contradictory with regard to the sign of pitch deviations observed. We make an attempt to explain the observations mentioned by (1) considering a number of factors involved, (2) comparing Platt and Racine's data with predictions made by the virtual-pitch theory (Terhardt, Stoll, \& Seewann, 1982a, 1982b), and (3) describing some related experimental data that we recently achieved with piano tones.

\section{Possible Factors Involved}

First it should be kept in mind that the effects are so small that it is appropriate to consider them in units of just noticeable differences (jnds) of pitch. For example, the "constant error" reported by Platt and Racine (1985) in their Figure 2 maximally extends from +6 cents to -12 cents. That pitch interval corresponds to about $1 / 5$ of a semitone and covers roughly 2 to 5 jnds. Although there is no doubt that pitch effects of that size can in principle be measured, it must be taken into account that there are a number of pronounced influences that inevitably will produce random variability.

With regard to evaluation of those influences, it is helpful to note that the virtual pitch of a complex tone is entirely dependent on spectral pitches of its components. Therefore, any pitch deviation observed with a complex tone can be reduced to corresponding deviations of its

The authors' mailing address is Institute for Electroacoustics, Technical University München, Arcisstrasse 21, D-8000 Munich 2, West Germany. spectral components. To evaluate pitch deviations of complex tones of the type reported by Platt and Racine, it is thus appropriate to begin by considering the following influences on the pitch of individual pure tones.

1. Quasi-random departures (from a smooth function) of the frequency-to-pitch characteristic of each particular ear of any particular subject: Those departures are intraindividually quite consistent, interindividually different, and can be demonstrated mainly by binaural diplacusis measurements and by octave matches (cf. van den Brink, 1975, 1977).

2. The influence of partial masking: The pitch of an individual pure tone with a fixed frequency in general may be shifted slightly up or down, depending on an additional sound signal superimposed as a "masker." In the present context, mutual partial masking of spectral components of a complex tone is a relevant example. The magnitude of this effect is interindividually different. Moreover, the resulting pitch shifts of components are dependent on auditory frequency selectivity, and this is interindividually different as well.

3. The influence of temporal envelope: The pitch of pure as well as of complex tones is significantly changed when a "percussive" temporal envelope is imposed (cf. Houtsma \& Rossing, 1987). Probably this effect is interindividually different.

4. The influence of sound pressure level (SPL): Generally it can be said that with increasing SPL, low pure tones get lower in pitch whereas high tones get higher (cf. Stevens, 1935; Terhardt, 1974; Verschuure \& van Meeteren, 1975). This effect is interindividually different as well.

Probably the above list of phenomena provides a fairly comprehensive outline of the effects that essentially are responsible for experimentally observed pitch deviations; and it is, in particular, the interindividual variance of the 
effects that explains a lot of the apparent discrepancies between results of different experiments. To get pitch matches that are reliable and of general validity within an interval of the order of 10 cents, the interindividual differences must be averaged out to a high extent and the physical parameters (e.g., SPL and spectral shape) of the audio signal present at the listener's eardrum must be known with a precision of a few decibels. It is in that sense that the possibility for drawing from experimental data quantitative conclusions of general validity on the basis of effects having an amount of only a few cents is seriously limited.

\section{Platt and Racine's Data Compared With the Virtual-Pitch Theory}

To evaluate and illustrate those limitations, we have made some calculations with the virtual-pitch model (cf. Terhardt et al., 1982a, 1982b). In Figure 1, the results of Platt and Racine's Experiment 1 are compared with predictions of the theory. The ordinate represents the frequency deviation (in cents) for equal pitch between a harmonic complex tone (75 $\mathrm{dB}$ SPL) and a pure tone (72 $\mathrm{dB}$ ); a positive deviation indicates that the pure tone is perceived as higher in pitch than the complex tone. Crosses show the mean results of "tuners" and "nontuners" com-

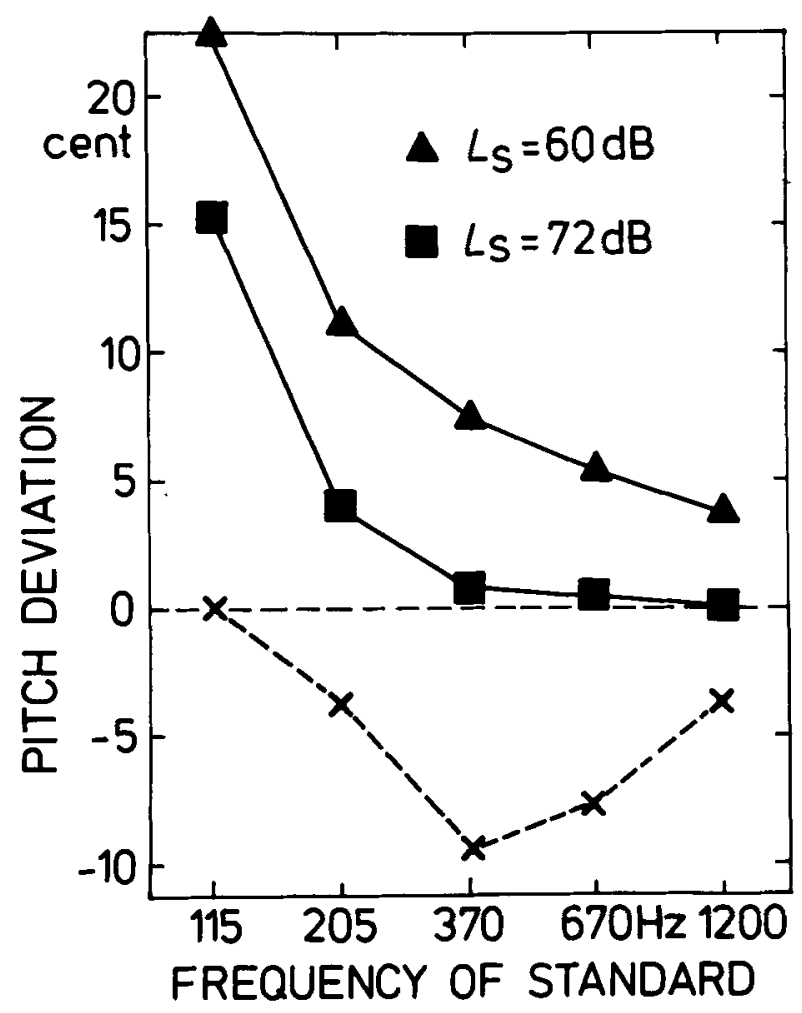

Figure 1. Predicted and experimental pitch deviations, as a function of frequency. The ordinate shows the pitch deviation between a pure tone and a harmonic complex tone (75 dB SPL). Positive deviations indicate that the pure tone is perceived as higher in pitch than the complex tone. Crosses: mean experimental results of Platt and Racine (1985). Triangles: prediction of the virtual pitch model, puretone SPL $60 \mathrm{~dB}$. Squares: pure-tone SPL $72 \mathrm{~dB}$.

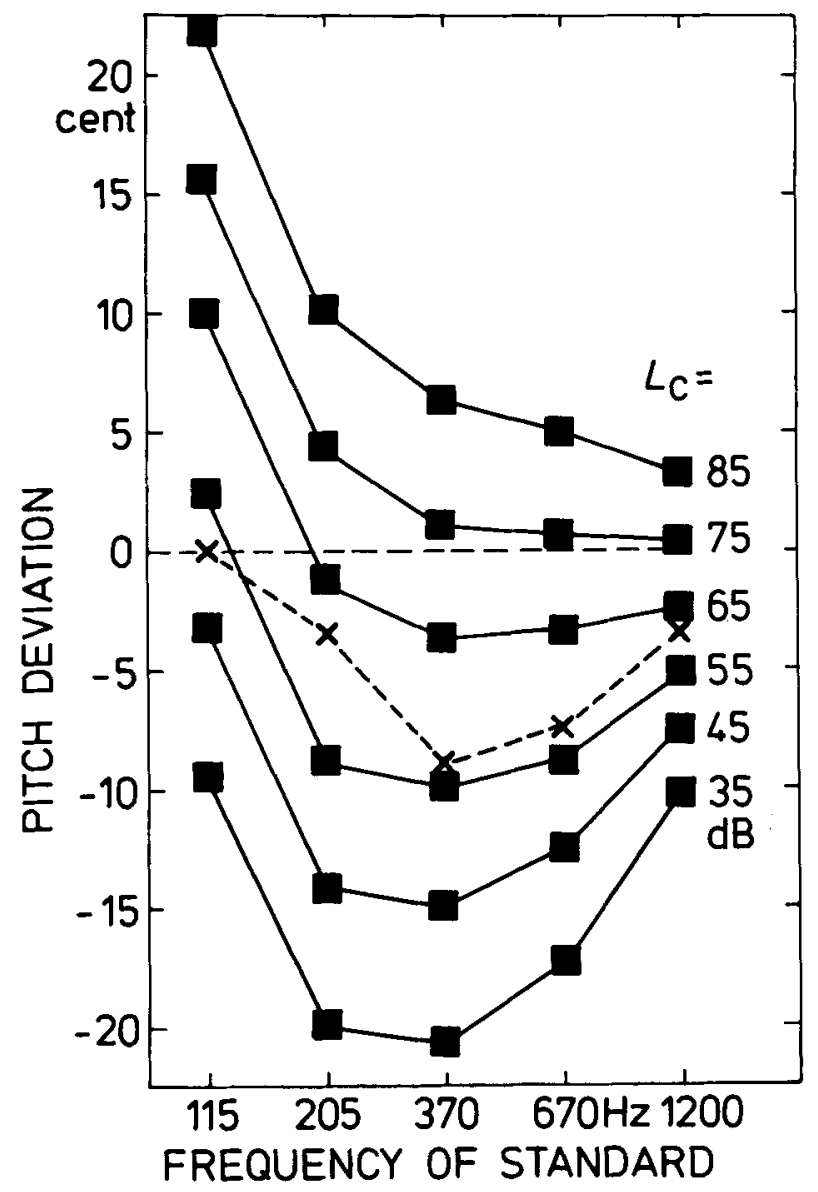

Figure 2. Theoretical evaluation of the influence of the complex tone's SPL (squares). Crosses: experimental data by Platt and Racine (1985), as in Figure 1. Coordinates are as in Figure 1.

bined, according to the data reported by Platt and Racine. ${ }^{1}$ Those data were obtained with a pure-tone SPL of $72 \mathrm{~dB}$. With the same SPL, the theory yields the results indicated by squares. For comparison, the theoretical "standard pitch" is also shown (triangles); this is defined as the pitch-equivalent frequency of a pure tone with $60 \mathrm{~dB}$ SPL. Figure 1 illustrates that the theory's prediction about shape, as a function of frequency, is about the same as that shown by the experimental data, although there is a discrepancy in that the theory predicts the pure tone to be higher in pitch than the complex tone, whereas the experiment indicates the opposite. One can see, however, that properly taking into account the SPLs of both the complex and the pure tone significantly shifts the theoretical prediction toward the experimental data.

With respect to the same set of experimental data as was used in Figure 1, Figure 2 illustrates the influence of the complex tone's SPL, symbolized $L_{c}$. Crosses again show Platt and Racine's data as explained above; squares show the theoretical results calculated with $72-\mathrm{dB}$ puretone SPL and the complex-tone SPLs indicated. Although there is remarkably good agreement of experimental and theoretical shapes, a distinct offset can be seen such that 
the experimental data (obtained with $75 \mathrm{~dB}$ SPL of the complex tone) are optimally represented by theoretical data pertinent to about $60 \mathrm{~dB}$. We do not conclude, of course, that Platt and Racine's SPL specifications are wrong by such a large amount. Possibly it is both some further parameter adjustment of the theory and higher precision of aurally effective SPL that would remedy the difference. We do not yet see a sufficient basis for making a final decision on that question.

Because we were unable to find useful specifications of the earphones' frequency response in Platt and Racine's paper, we made an evaluation of the possible influence of that factor, again using the virtual-pitch model. We compared the pitch, theoretically obtained with an entirely flat earphone response, with that pertinent to the response shown in the inset of Figure 3. (The latter is typical of the Beyer DT48 earphone without an equalizer.) In Figure 3, crosses once again show Platt and Racine's experimental data; squares correspond to a flat frequency response, and triangles to that shown in the inset. It turns out that, although spectral composition of the stimulus is, of course, dependent on the earphone response, the details of a reasonable earphone's frequency response are more or less irrelevant with respect to the pitch-matching effects discussed here. It is essentially the SPL of the pure and/or the complex tone that must be regarded as respon-

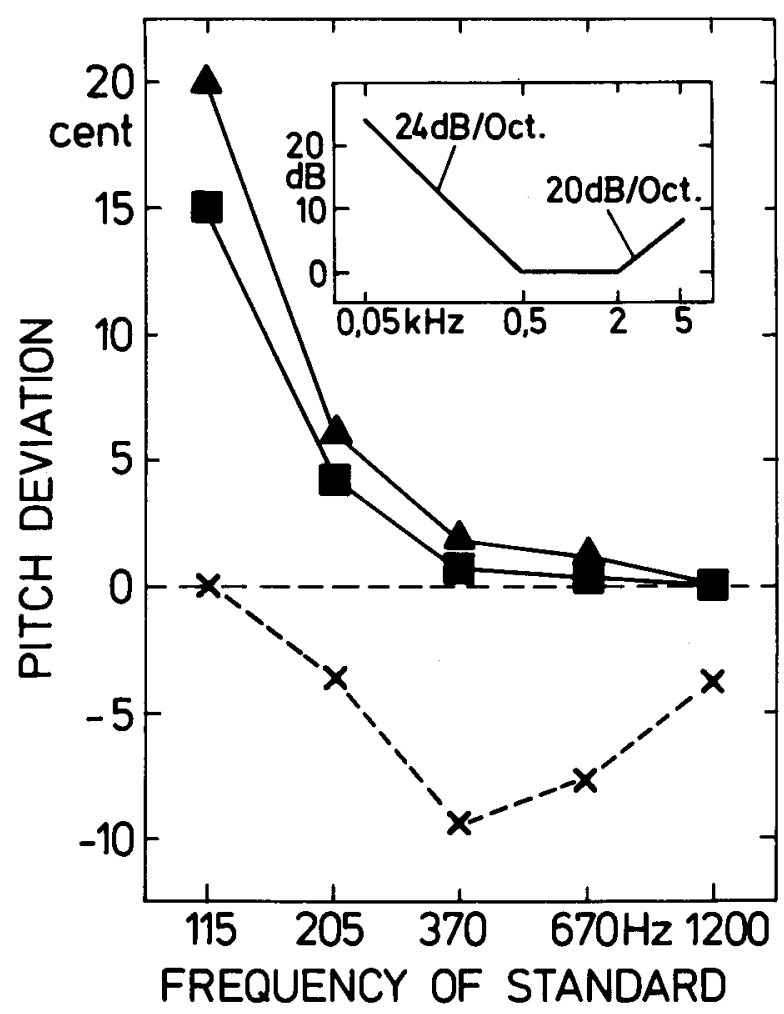

Figure 3. Theoretical evaluation of the influence of the earphone's frequency response. Squares: flat response, such as assumed in Figure 1. Triangles: frequency response such as that of Beyer DT 48 without equalizer (inset). Crosses: as in previous figures.

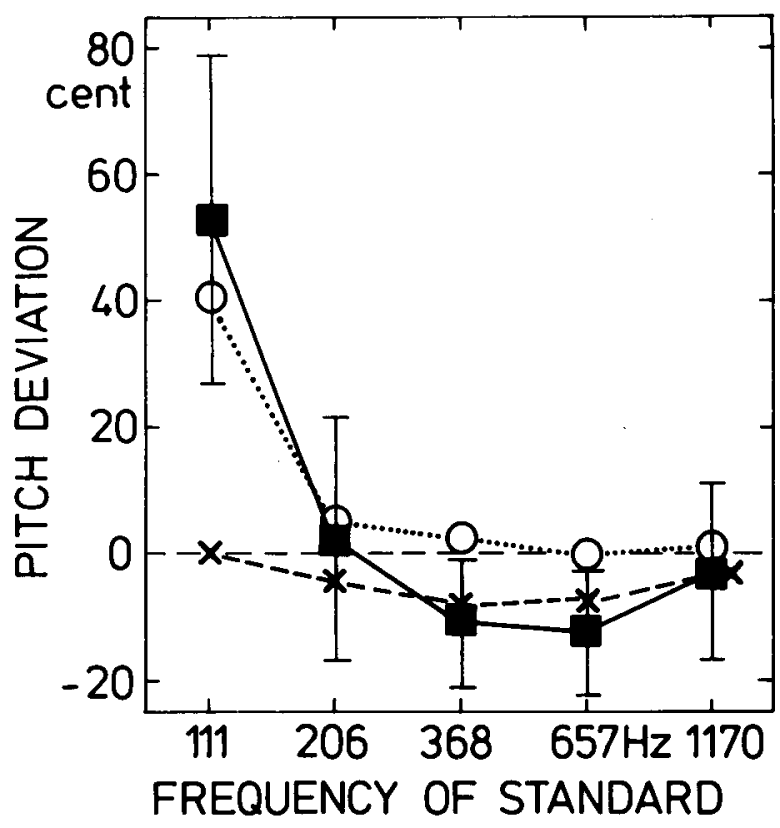

Figure 4. Pitch deviations of five piano tones. Squares: experimental results; vertical bars indicate confidence interval (95\% level). Circles: theoretical prediction. Crosses: as in previous figures. ${ }^{2}$

sible for the apparent discrepancies observed in different experiments by different authors.

\section{Pitch Matches of Piano Tones}

In our experiments, 10 subjects were asked to match a pure tone in pitch to one of the 88 tones of the piano scale, one after the other in random order. Our concern was not primarily precision of pitch matching (i.e., the aspect of tuning), but rather that of getting a "pitch scale" of a piano, as opposed to its "frequency scale." This is why we were more concerned about getting just a sufficient number of matches to each of the piano tones than about getting many matches to only a few tones. Each subject made two matches to every piano tone. SPL of pure matching tone was $70 \mathrm{~dB}$, and that of piano tones was as close as possible to $70 \mathrm{~dB}$. Duration of each tone, and the pause between piano and matching tones, was $1 \mathrm{sec}$. In spite of the physical and methodological differences (which are small anyhow), it is elucidatory to compare the results with those of Platt and Racine.

Figure 4 shows the results pertinent to five piano tones selected from our data (filled squares). ${ }^{2}$ The ordinate scales the frequency deviation between the lowest partial of the piano tone, extracted by spectrum analysis, and the pure matching tone, for equal pitch. As in the previous figures, positive ordinate values indicate that the pure tone is higher in pitch than the complex tone. As a function of frequency, the data show the same shape as discussed before, and the pitch deviations obtained by the virtualpitch theory (open circles) are in remarkable agreement. ${ }^{3}$ Comparison of the entire patterns of experimental and theoretical results, including Platt and Racine's (crosses), 
reveals that actually there is more agreement than disparity between the data sets.

\section{Conclusions}

The present study, based on and stimulated by Platt and Racine's (1985) data, illustrates that a general statement such as "a harmonic complex tone is higher in pitch than a pure tone having the same frequency" is as insufficient and inadequate as would be the opposite statement. To get valuable data on the precise pitch of complex tones, considerable interindividual differences must be averaged out. The effects remaining are strongly affected by SPL and frequency of the stimuli. The theory of virtual pitch turns out to be quite helpful in gaining greater insight into those interdependencies. And since it is second-order effects that are discussed here, the theory provides remarkable explanations and suggestions for further research. Without a question, further careful investigations are necessary, as several details concerning those secondorder pitch effects still are not sufficiently explained.

\section{REFERENCES}

Houtsma, A. J. M., \& Rossing, T. D. (1987). Effects of signal envelope on the pitch of short complex tones. Journal of the Acoustical Society of America, 81, 439-444.

Platt, J. R., \& Racine, R. J. (1985). Effect of frequency, timbre, experience, and feedback on musical tuning skills. Perception \& Psychophysics, 38, 543-553.

STEVENS, S. S. (1935). The relation of pitch to intensity. Journal of the Acoustical Society of America, 6, 150-154.
Terhardt, E. (1974). Pitch of pure tones: Its relation to intensity. In E. Zwicker \& E. Terhardt (Eds.), Facts and models in hearing (pp. 353-360). Heidelberg: Springer.

Terhard, E., Stoll, G., \& Seewann, M. (1982a). Algorithm for extraction of pitch and pitch salience from complex tonal signals. Journal of the Acoustical Society of America, 71, 679-688.

Terhardt, E., Stoll, G., \& Seewann, M. (1982b). Pitch of complex signals according to virtual-pitch theory: Tests, examples, and predictions. Joumal of the Acoustical Society of America, 71, 671-678. VAN DEN BRINK, G. (1975). The relation between binaural diplacusis for pure tone and for complex sounds under normal conditions and with induced monaural pitch shifts. Acustica, 32, 160-165.

VAN DEN BRINK, G. (1977). Octave and fifth settings for pure tones and residue tones. In E. F. Evans \& J. P. Wilson (Eds.), Psychophysics and physiology of hearing (pp. 373-379). London: Academic Press.

Verschuure, J., \& van Meeteren, A. A. (1975). The effect of intensity on pitch. Acustica, 32, 33-44.

\section{NOTES}

1. Since the theory was designed to quantitatively represent the mean behavior of normally hearing listeners (i.e., not just tuners, for instance), we compare its predictions with the means of both tuners and nontuners.

2. The piano tones were recorded in a studio using a Schimmel upright piano that had just been tuned. The frequencies shown at the abscissa of Figure 4 naturally depart slightly from those used in the Platt and Racine experiments, since our experiment was carried out independently.

3. Since it is second-order effects of pitch that are being considered, the agreement may indeed be called "remarkable."

(Manuscript received January 20, 1987; revision accepted for publication June $30,1987$. 